\title{
Biomechanics, diagnosis, and treatment outcome in inflammatory myopathy presenting as oropharyngeal dysphagia
}

\author{
R B Williams, M J Grehan, M Hersch, J Andre, I J Cook
}

See end of article for authors' affiliations

Correspondence to: Associate Professor I J Cook, Department of Gastroenterology, St George Hospital, Gray St, Kogarah, New South Wales, Australia 2217; I.Cook@unsw.edu.au

Accepted for publication 13 September 2002

\begin{abstract}
Aims: In patients with inflammatory myopathy and dysphagia, our aims were to determine: (1) the diagnostic utility of clinical and laboratory indicators; (2) the biomechanical properties of the pharyngo-oesophageal segment; (3) the usefulness of pharyngeal videomanometry in distinguishing neuropathic from myopathic dysphagia; and (4) clinical outcome.

Methods: Clinical, laboratory, and videomanometric assessment was performed in 13 patients with myositis and dysphagia, in 17 disease controls with dysphagia (due to proven CNS disease), and in 22 healthy age matched controls. The diagnostic accuracy of creatine kinase (CPK), erythrocyte sedimentation rate, antinuclear antibody, and electromyography (EMG) were compared with the gold standard muscle biopsy. The biomechanical properties of the pharyngo-oesophageal segment were assessed by videomanometry.

Results: Mean time from dysphagia onset to the diagnosis of myositis was 55 months (range 1-180). One third had no extrapharyngeal muscle weakness; $25 \%$ had normal CPK, and EMG was unhelpfu in $28 \%$. Compared with neurogenic controls, myositis patients had more prevalent cricopharyngeal restrictive disorders $(69 \%$ v $14 \% ; p=0.0003)$, reduced upper oesophageal sphincter (UOS) opening $(p=0.01)$, and elevated hypopharyngeal intrabolus pressures $(p=0.001)$. Videomanometric features favouring a myopathic over a neuropathic aetiology were: preserved pharyngeal swallow response, complete UOS relaxation, and normal swallow coordination. The 12 month mortality was $31 \%$.

Conclusions: The notable lack of supportive clinical signs and significant false negative rates for laboratory tests contribute to the marked delay in diagnosis. The myopathic process is strongly associated with restricted sphincter opening suggesting that cricopharyngeal disruption is a useful adjunct to immunosuppressive therapy. The condition has a poor prognosis.
\end{abstract}

A broad spectrum of disorders are potentially responsible for neurogenic dysphagia without obvious cause. Inflammatory myopathy should be considered in any patient with otherwise unexplained oropharyngeal dysphagia. One quarter of patients with inflammatory myopathy have dysphagia at presentation ${ }^{2}$ and dysphagia occurs in up to $60 \%$ of cases at some time during the illness. ${ }^{3-8}$ On the other hand, there is very little published data on the prevalence of inflammatory myopathy in dysphagic patients, their treatment outcomes and prognosis, and the utility of laboratory tests for diagnosis in this setting. In our experience, the diagnosis of myopathy in patients presenting with oropharyngeal dysphagia can be elusive. Correct diagnosis is particularly important in this population as the underlying systemic condition is treatable in its own right. Further, limited evidence suggests some affected patients respond to cricopharyngeal disruption by either dilatation ${ }^{9}$ or myotomy. ${ }^{3-6}$ This observation suggests that myositis might be associated with restriction of upper oesophageal sphincter (UOS) opening similar to that observed with a cricopharyngeal bar ${ }^{10}$ or a Zenker's diverticulum, ${ }^{11}{ }^{12}$ but this hypothesis has not been systematically examined. ${ }^{6}$

In a group of patients with myositis causing oralpharyngeal dysphagia, our aims were to: (1) evaluate the diagnostic utility of clinical and laboratory parameters in the diagnosis of inflammatory myopathy; (2) compare swallow biomechanics of the pharynx and upper oesophageal sphincter (UOS) with healthy controls and disease controls with a proven neurogenic cause for dysphagia; (3) examine temporal relationships among events during the swallow to determine the diagnostic utility, if any, of combined pharyngeal manometry and videoradiography in distinguishing myopathic from neurogenic causes of oropharyngeal dysphagia; and (4) assess the clinical outcome and response to cricopharyngeal disruption by either cricopharyngeal myotomy or dilatation.

\section{METHODS}

\section{Patients and controls}

Patients $(n=13)$ were retrieved from a database of 529 patients with oropharyngeal dysphagia which had been prospectively collected between 1991 and 1998 in a tertiary referral centre (table 1). The diagnosis of inflammatory myopathy was confirmed by the finding of abnormalities in two or more of the following: raised serum creatine kinase (CPK), electromyography (EMG), or muscle biopsy. Subclassification of cases was based on the characteristic histological changes on limb muscle biopsy of polymyositis, dermatomyositis, or inclusion body myositis. Cricopharyngeal muscle histopathology was available in three of four patients who underwent cricopharyngeal myotomy.

Disease controls ( $\mathrm{n}=17,12$ male; mean age 74 years (range 58-90)) were those with a proven central neurogenic cause of oropharyngeal dysphagia, and who had undergone a CPK estimation and either a videoswallow or videomanometric

Abbreviations: UOS, upper oesophageal sphincter; $C P$, cricopharyngeus; EMG, electromyography; CPK, creatine kinase; ANA, antinuclear antibody; ESR, erythrocyte sedimentation rate; PEG, percutaneous gastrostomy. 
Table 1 Demographic and clinical findings

\begin{tabular}{|c|c|c|c|c|c|c|c|c|c|c|}
\hline Subject & Sex & $\begin{array}{l}\text { Age } \\
\text { (y) }\end{array}$ & Final diagnosis & $\begin{array}{l}\text { Duration of } \\
\text { dysphagia prior to } \\
\text { evaluation } \\
\text { (months) }\end{array}$ & $\begin{array}{l}\text { Presentation to } \\
\text { diagnosis of cause of } \\
\text { dysphagia (months) }\end{array}$ & $\begin{array}{l}\text { Head and neck } \\
\text { muscle } \\
\text { involvement }\end{array}$ & $\begin{array}{l}\text { Muscle } \\
\text { weakness }\end{array}$ & Aspiration & $\begin{array}{l}\text { Dysphagia } \\
\text { severity }\end{array}$ & $\begin{array}{l}\text { UOS } \\
\text { restrictive } \\
\text { feature }\end{array}$ \\
\hline \multicolumn{11}{|c|}{ Idiopathic presentation } \\
\hline 1 & $\mathrm{~F}$ & 81 & Polymyositis & 180 & 0.46 & No & No & No & Mild & Yes \\
\hline 2 & $\mathrm{~F}$ & 59 & Polymyositis & 72 & 11 & No & No & Yes & Mild & Yes \\
\hline 3 & $\mathrm{~F}$ & 74 & Polymyositis & 24 & 0.23 & Yes & Proximal & Yes & Severe & Yes \\
\hline 4 & $\mathrm{~F}$ & 67 & Polymyositis & 6 & 0.23 & No & Proximal & Yes & Moderate & No \\
\hline 5 & $M$ & 69 & Polymyositis & 36 & 45 & No & No & Yes & Mild & Yes \\
\hline 6 & $M$ & 75 & Interstitial myositis & 11 & 0.46 & Yes & Yes & Yes & Severe & No \\
\hline 7 & $M$ & 74 & Polymyositis & 19 & 0.7 & Yes & Proximal & No & Mild & Yes \\
\hline 8 & $\mathrm{~F}$ & 88 & Polymyositis & 144 & 0.06 & Yes & Proximal & Yes & Mod-sev & Yes \\
\hline 9* & $\mathrm{F}$ & 22 & Polymyositis & 1 & 0.23 & No & Proximal & No & - & No \\
\hline \multicolumn{11}{|c|}{ Confirmed myopathy at time of referral } \\
\hline 10 & M & 45 & $\begin{array}{l}\text { Inclusion body } \\
\text { myositis }\end{array}$ & 18 & Prior 4 months & Yes & $\begin{array}{l}\text { Proximal } \\
\text { limb }\end{array}$ & No & Mild & Yes \\
\hline 11 & $M$ & 71 & Dermatomyositis & 16 & Prior 10 years & No & $\begin{array}{l}\text { Proximal } \\
\text { limb }\end{array}$ & Yes & Mild & No \\
\hline 12 & $\mathrm{~F}$ & 86 & Polymyositis & 24 & Prior 4 years & No & $\begin{array}{l}\text { Proximal } \\
\text { limb }\end{array}$ & No & Mild-Mod & Yes \\
\hline 13 & $\mathrm{~F}$ & 74 & Polymyositis & 36 & 0.46 & Yes & $\begin{array}{l}\text { Proximal, } \\
\text { facial }\end{array}$ & Yes & Severe & Yes \\
\hline
\end{tabular}

study. This meant excluding those with a peripheral neuropathic or myopathic aetiology. Hence patients with disorders potentially affecting the lower motor neurone (motor neurone disease, head and neck malignancy), neuromuscular junction (myasthenia), or which might affect the cricopharyngeus muscle (non-inflammatory myopathies), or those with demonstrated failure of manometric UOS relaxation ${ }^{13}$ were excluded from the control group because a comparison group for videomanometry was required. Dysphagia was due to cerebrovascular accident in 12, Parkinson's disease in four, and multiple sclerosis in one. Two patients with CPK measured postoperatively or after myocardial infarction were excluded from analysis of laboratory parameters.

Healthy aged individuals $(n=22$; mean age 68 (10) years) also served as controls for radiographic and manometric measures.

\section{Clinical assessments}

Oropharyngeal dysphagia was defined as difficulty with the act of swallowing together with one or more of the following deglutitive symptoms: bolus holdup; multiple swallows required to clear the pharynx; deglutitive coughing and/or choking; or postnasal regurgitation. ${ }^{14} \mathrm{~A}$ global assessment of dysphagia severity was made clinically by one of the authors (IJC) and was classified on a five point scale as mild (amenable to slight dietary modification), mild-moderate (dysphagia requiring dietary modification and with an increased time to complete a meal), moderate (dietary modification and minor symptoms of aspiration), moderate-severe (marked dietary modification required together with moderate aspiration symptoms), and severe (severe dysfunction with aspiration sufficient to necessitate introduction of non-oral feeding).$^{14}$

The diagnostic sensitivity and specificity of the laboratory studies was determined in the subgroup of biopsy proven inflammatory myopathy cases that were undiagnosed at presentation with dysphagia $(n=8)$. An elevated CPK was defined as greater than the upper limit of normal for our laboratory $(200 \mathrm{U} / \mathrm{l})$. An antinuclear antibody (ANA) titre greater than $1: 160$ and erythrocyte sedimentation rate (ESR) above 20 were the cut off levels considered abnormal. The EMG findings of the investigating neurologist were classified as positive if the findings were considered to be suggestive of myopathy as the principal abnormality, or negative if the study was normal, non-specific, or suggested an alternative diagnosis.
Videofluoroscopic studies were reviewed and assessed qualitatively for the following. A restrictive abnormality was defined as the presence of a cricopharyngeal (CP) bar or stenosis. ${ }^{15}$ A CP bar was defined as a persistent post-cricoid indentation seen in the lateral projection only. A CP stenosis was defined as a circumferential constriction in the postcricoid region which was apparent in both anteroposterior and lateral planes.

Clinical outcome was assessed 12 months after presentation in patients with inflammatory myopathy. The outcomes of interest included: (a) need for non-oral (usually percutaneous gastrostomy (PEG)) feeding; (b) pneumonia; and (c) death. $\mathrm{CP}$ dilatation or myotomy was performed as clinically indicated and the response was assessed subjectively and objectively one and six months post procedure. A subjective improvement was considered to be present if the patient described the improvement in overall swallowing function as "very good or excellent". An objective response was defined as weight gain $>1 \mathrm{~kg}$ in the absence of enteral feeding. Responses were considered sustained if the benefits were present six months post procedure.

\section{Combined videoradiography and pharyngeal manometry}

Swallowing function was studied by simultaneous videoradiography and manometry as previously described..$^{14} 16$ Both control groups and the patient group were studied using the same standard protocol. Briefly, images of barium swallows were recorded in the lateral and anteroposterior projections using a 9" Toshiba (Kawasaki, Japan) image intensifier. Fluoroscopic images were recorded on videotape at 25 frames per second by a VHS video recorder (Panasonic, AG6500, Osaka, Japan) for later analysis. The correction factor for magnification was determined prior to each study by placing two metallic markers set $3 \mathrm{~cm}$ apart in the field of the image intensifier, above the subject's head but in the plane of the UOS. Subjects swallowed, as tolerated (depending on aspiration, if present), duplicate $2,5,10$, and $20 \mathrm{ml}$ boluses of high density liquid barium suspension (250\% (wt/vol), E-Z-HD, E-Z EM Inc., Westbury, New York, USA). If any more than minor aspiration occurred with a specific bolus volume, larger volumes were not attempted. Included in the field of view in the lateral projection were the incisor teeth anteriorly, hard 


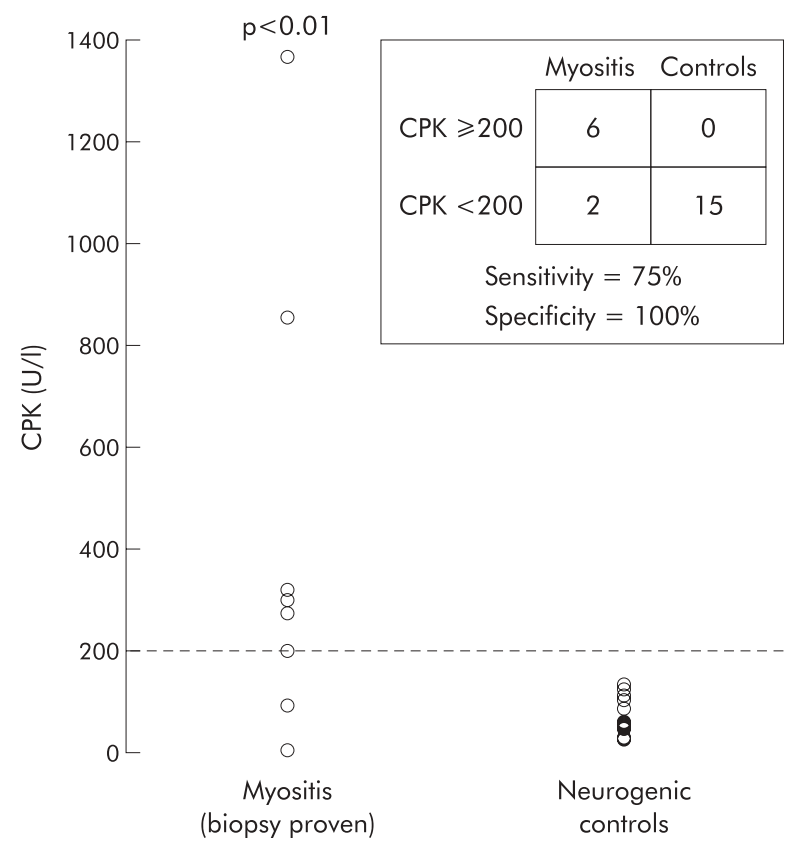

Figure 1 Serum creatine kinase (CPK) levels in the subset of patients with biopsy proven myositis $(n=8)$ and neurogenic controls with oropharyngeal dysphagia. Note that two of eight myositis patients in this subset had normal CPK levels.

palate superiorly, cervical spine posteriorly, and proximal cervical oesophagus inferiorly.

Pharyngeal pressures were measured using both perfusion and solid state manometric techniques. Initially we passed transnasally a 9 lumen (OD $6 \mathrm{~mm}$; ID each lumen $0.5 \mathrm{~mm}$ ) silastic/polyvinyl chloride manometric catheter, incorporating five perfused sideholes spaced at $1.5 \mathrm{~cm}$ intervals and a $6 \mathrm{~cm}$ sleeve assembly (Dentsleeve, Wayville, South Australia). The sleeve was positioned to straddle the UOS to accommodate the axial mobility of the sphincter. The sleeve sensor had a $5 \times 3$ $\mathrm{mm}$ oval cross section to maintain its anteroposterior orientation within the UOS. Five perfused sideholes proximal to the sleeve, with the most distal sidehole in the pharynx at the level of the proximal sleeve margin, recorded pharyngeal pressures. A sidehole located $3 \mathrm{~cm}$ distal to the proximal sleeve margin, in midsleeve position, aided positioning the sleeve such that its midpoint was in the centre of the UOS high pressure zone at rest. The sleeve assembly and sideholes were perfused by a low compliance pneumohydraulic perfusion system at 0.6 $\mathrm{ml} / \mathrm{min}$. The sideholes were only perfused while swallows were being recorded to avoid fluid accumulation in the pharynx. Pharyngeal and UOS pressures detected by the perfused catheter were registered by external transducers (Spectramed Medical Products, Singapore) and all signals were amplified and acquired at $200 \mathrm{~Hz}$ per channel (GastroMac, Neomedix Systems, Sydney, Australia) on a Macintosh computer (Apple, Cupertino, California, USA) using Gastromac software (Neomedix Systems). All pressures were referenced to basal hypopharyngeal pressure. A purpose built video digital timer unit (Practel Sales International, Holden Hill, South Australia) imprinted simultaneously the elapsed time on the video images in hundredths of seconds and a signal on the pressure tracing each whole second to permit precise correlation of video images with pharyngeal pressures.

If peak pharyngeal pressures exceeded $60 \mathrm{~mm} \mathrm{Hg}$, a separate catheter with five solid state transducers, spaced at $1.5 \mathrm{~cm}$ intervals (Gaeltec, Dunvegan, Isle of Skye, UK), was used to make repeat measures of peak pharyngeal pressures following removal of the perfused catheter. ${ }^{14}$ This catheter was passed transnasally and the posterior orientation of the transducers was readily verified radiographically. The catheter was positioned such that the distal transducer lay just proximal to the UOS to capture the UOS pressure profile during maximal deglutitive ascent of the sphincter. In this position the middle transducer, recording mid pharyngeal pressures, lay at the level of the valleculae.

\section{Videomanometric data analysis}

Maximal UOS dimensions during sphincter opening were measured fluoroscopically, for a range of bolus volumes, in the sagittal plan in all patients and in the transverse plane in those patients in whom sufficient swallows were tolerated before significant aspiration precluded further swallows. Extent of UOS opening was classified as "normal" if the maximal sagittal diameter lay within the normal laboratory range (mean \pm 2 SD for healthy controls), "partial" if opening was observed but maximal sagittal diameter fell below the lower limit of normal ( $<6 \mathrm{~mm}$ for $2 \mathrm{ml}$ barium bolus), and was "absent" if no contrast penetrated the sphincter zone during attempted deglutition. UOS opening duration was similarly classified as normal, partial $(<0.31 \mathrm{~s})$, or absent.

\section{Manometric measures and definitions}

UOS pressures were referenced to resting pre-swallow hypopharyngeal pressure. Basal UOS pressure was determined by averaging end expiratory UOS pressure over a one minute period interval 10 minutes after catheter placement to permit subject adaptation. UOS relaxation was assessed solely on manometric criteria while UOS opening was defined on radiological criteria (see above). The adequacy of UOS relaxation was determined from the nadir UOS pressure measured during dry swallows, prior to administration of any test boluses. Deglutitive UOS relaxation was classified as failed if nadir UOS pressure during $100 \%$ of dry swallows exceeded $10 \mathrm{~mm} \mathrm{Hg}$ in individuals $\geqslant 55$ years of age, or exceeded $13 \mathrm{~mm}$ $\mathrm{Hg}$ in individuals $<55$ years of age. ${ }^{13} 141718$

The recording site, from which "mid pharyngeal" pressure was measured, lay $4.5 \mathrm{~cm}$ proximal to the mid point of the UOS at the apogee of its upward deglutitive motion. Hypopharyngeal intrabolus pressure was measured at the perfused sidehole immediately proximal to the UOS at the proximal margin of the sleeve in controls and in all patients in whom some trans-sphincteric bolus flow was observed radiologically. Hypopharyngeal intrabolus pressure was defined, on the basis of correlation of manometry and fluoroscopy, as the pressure registered at the time point midway between the arrival of the bolus head and the departure of the bolus tail at that site. ${ }^{116}$ In instances where there was a dwell time by the bolus head at the hypopharyngeal sidehole $\geqslant 100 \mathrm{~ms}$, mid intrabolus pressure was estimated from the time point midway between the onset and termination of that particular instance of trans-sphincteric bolus flow. All manometric measures were made unblinded.

\section{Temporal measures}

We conceptualised "swallow coordination" using three temporal measures of important events in the swallow sequence (time of onset of UOS relaxation, time of UOS opening, and time of UOS closure), referenced to the time of initial movement of the tongue tip against the posterior surface of the maxillary incisors, an event that marks the onset of the oral phase of swallowing. ${ }^{11}{ }^{14} 16$ We compared the timing of these three events in patients with myositis and neurogenic controls with previously defined normal ranges for these quantities, estimated from our aged controls..$^{17} 17$ We sorted each temporal interval into $0.2 \mathrm{~s}$ bins for patients and controls, and examined the frequency distributions of the three time intervals of primary interest. We hypothesised that the myopathic swallow (unless severely deranged) should demonstrate normal coordination while the swallow deranged from neurogenic causes is more likely to show incoordination. 

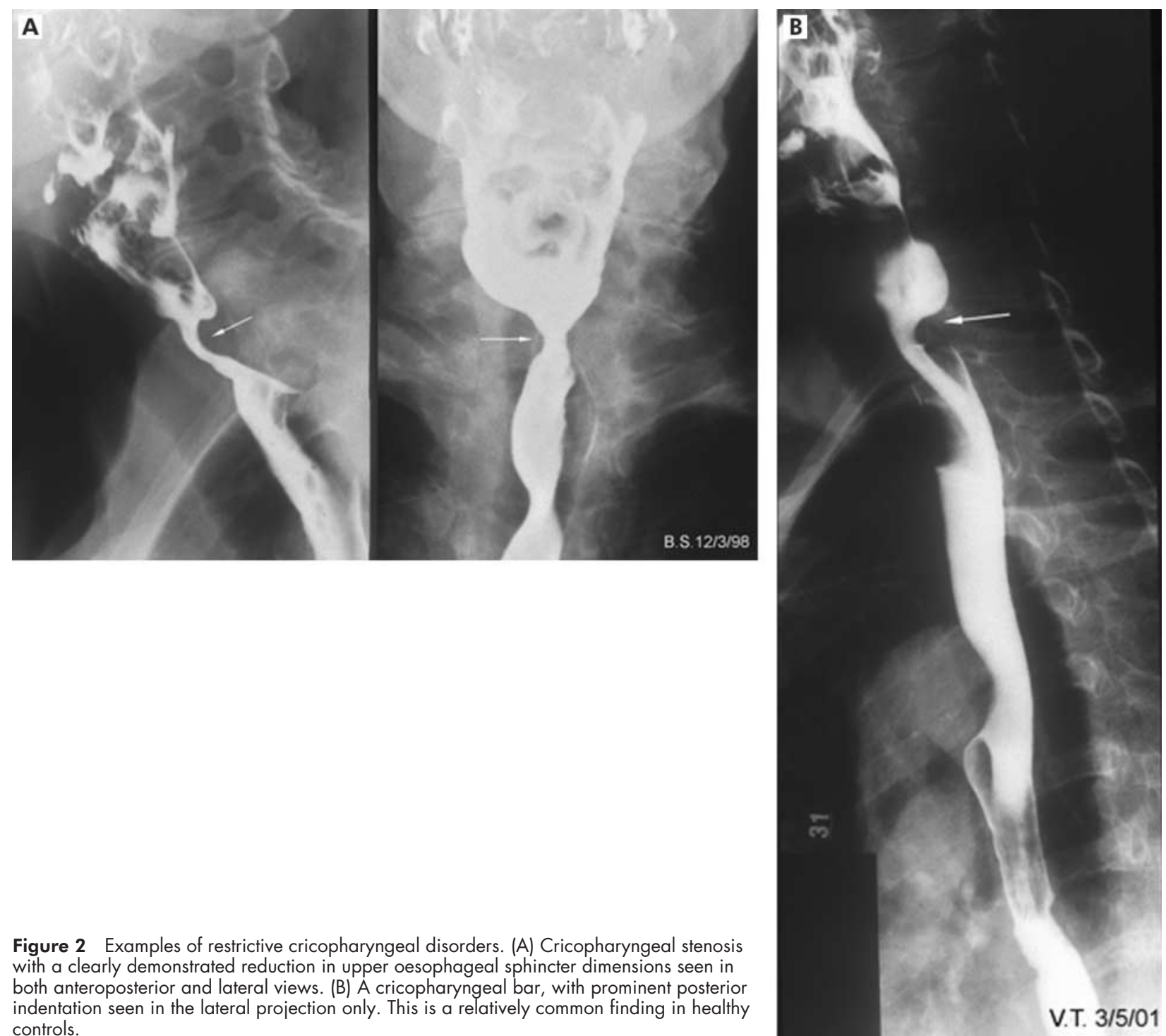

Figure 2 Examples of restrictive cricopharyngeal disorders. (A) Cricopharyngeal stenosis with a clearly demonstrated reduction in upper oesophageal sphincter dimensions seen in both anteroposterior and lateral views. (B) A cricopharyngeal bar, with prominent posterior indentation seen in the lateral projection only. This is a relatively common finding in healthy controls.

for three multiple comparisons. ${ }^{19}$ Duplicate values for each

\section{Statistical analysis}

All data are presented as mean (SEM) unless stated otherwise. A $\chi^{2}$ test was used to make inferences regarding differences between proportions within patients and controls with and without pharyngo-oesophageal segment abnormalities. Inferences on proportions of patients and controls with normal and abnormal ANA titres were made using the $\chi^{2}$ test. A MannWhitney $U$ test (non-parametric, unpaired data) was used to compare CPK and ESR values between patients and controls. Comparisons of basal UOS pressure among myositis patients and neurogenic and aged controls were made using an unpaired Student's $t$ test, corrected using a Bonferroni-Dunn procedure; alpha ( $p$ value) significant at $<0.0167$, correction

Table 2 Pharyngo-oesophageal segment radiographic abnormalities

\begin{tabular}{|c|c|c|c|c|}
\hline & \multicolumn{2}{|l|}{$\mathrm{CP}$} & \multirow[b]{2}{*}{ Zenker's } & \multirow{2}{*}{$\begin{array}{l}\text { Radiographic } \\
\text { abnormality }\end{array}$} \\
\hline & Bar & Stenosis & & \\
\hline Myositis ( $n=13$ ) & 4 & 5 & 3 & $69 \%$ \\
\hline Neurogenic controls ( $n=17$ ) & 0 & 1 & 0 & $6 \%$ \\
\hline Aged controls $(n=22)$ & 1 & 0 & 0 & $5 \%$ \\
\hline
\end{tabular}

${ }^{*} p<0.002$ versus neuorgenic controls; $p<0.001$ versus aged controls. $\mathrm{CP}$, cricopharyngeus. subject were averaged before calculation of group mean data for each bolus volume swallowed. Group mean data were compared among patients and controls using a two factor ANOVA. Although appropriate and with greater statistical power, a repeat measure ANOVA design was not adopted because there were some missing values (as not all patients could swallow the entire range of bolus volumes due to disease severity). ${ }^{19}$ For classification of pharyngeal peak pressures and temporal variables, we used data recorded for $2 \mathrm{ml}$ and $5 \mathrm{ml}$ barium boluses as these were the two most common volumes available in both myositis patients and neurogenic controls. A $\chi^{2}$ test was used to compare proportions of myositis patients and neurogenic controls that demonstrated swallow incoordination. Statistical calculations were performed using StatVIEW 4.5 (Abacus Concepts Inc, Berkerly, California, USA).

\section{RESULTS}

\section{Demographics and clinical features}

Thirteen patients (five males; mean age 68 years (range 22-88)) with inflammatory myopathy were identified; 11 with polymyositis and one each with dermatomyositis and inclusion body myositis (table 1). Mean time from the onset of dysphagia to the diagnosis of inflammatory myopathy was 55 months (range 1-180). In nine of 13 cases, dysphagia was the 
Table 3 Videomanometric findingst

\begin{tabular}{lccc}
\hline & Aged controls & Neurogenic controls & Myositis patients \\
\hline Absent pharyngeal swallow response & $0 / 22$ & $3 / 14$ & $0 / 13$ \\
Basal UOS pressure $(\mathrm{mm} \mathrm{Hg})$ & $45(5)$ & $25(4)^{*}$ & $38(4)$ \\
Nadir UOS pressure $(\mathrm{mm} \mathrm{Hg})$ & $2(1)$ & $3(2)$ & $3(2)$ \\
Peak pharyngeal pressure $(\mathrm{mm} \mathrm{Hg})$ & $124(12)$ & $91(14)$ & $71(7)$ \\
& - & $(3 / 14)$ & $(6 / 13)$ \\
\hline & & & \\
*p<0.05 versus aged controls. & & \\
† 14 of 17 neurogenic controls studied videomanometrically. \\
UOS, upper oesophageal sphincter.
\end{tabular}

presenting complaint prompting presentation, of whom one third had limb weakness, one third had limb and facial or bulbar weakness, and the remaining third had no detectable weakness. All four patients with a prior diagnosis of myopathy had proximal muscle weakness (table 1)

\section{Diagnostic utility of investigations}

The diagnostic utility of EMG, CPK, ANA, and ESR was assessed in the subgroup of eight cases presenting with no prior diagnosis of myopathy and who had muscle biopsy confirmation of inflammatory myopathy. For this purpose, muscle biopsy was considered the "gold standard" for myositis. Serum CPK was raised in 6/8 patients with biopsy proven inflammatory myopathy and in none of 15 controls. Serum CPK values were significantly elevated compared with controls $(p=0.008)$ (fig 1$)$. An elevated CPK had a sensitivity of $75 \%$ and specificity of $100 \%$ for the diagnosis of inflammatory myopathy. ESR was elevated in 3/8 cases and in 5/11 neurogenic controls (NS). ANA was positive in $4 / 8$ cases and in $2 / 8$ neurogenic controls (NS).

A

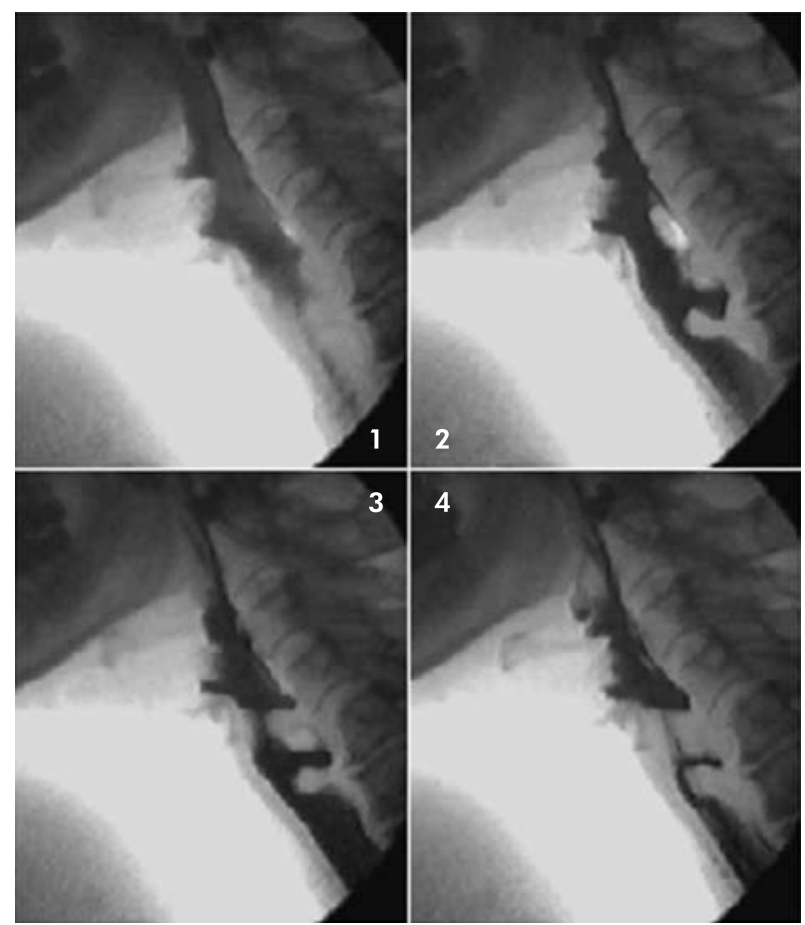

The initial EMG demonstrated unequivocal myopathic changes in 5/7 cases who had EMG (not done in 1/8). EMG was normal in one case and showed non-specific changes in one.

\section{Videoradiographic and manometric features}

Nine of 13 cases $(69 \%)$ had radiographically demonstrated restrictive pharyngo- oesophageal segment abnormalities (fig 2 ; table 2 ). This proportion was significantly higher than that seen $(1 / 17)$ in neurogenic controls $\left(p=0.0003, \chi^{2}\right.$ test $)$. Among myopathic patients with CP abnormalities, three had Zenker's diverticula while none was present in controls (fig 2). Aspiration was evident during videoradiography in $8 / 13$ (61\%) patients with myositis and in 7/17 (41\%) disease controls. In none of the myositis patients was the pharyngeal swallow response absent, but this radiographic abnormality was noted in 3/14 neurogenic controls who had undergone videomanometry.

Basal UOS pressure did not differ significantly between patients with myositis (38 (4) $\mathrm{mm} \mathrm{Hg}$ ) and aged controls (45

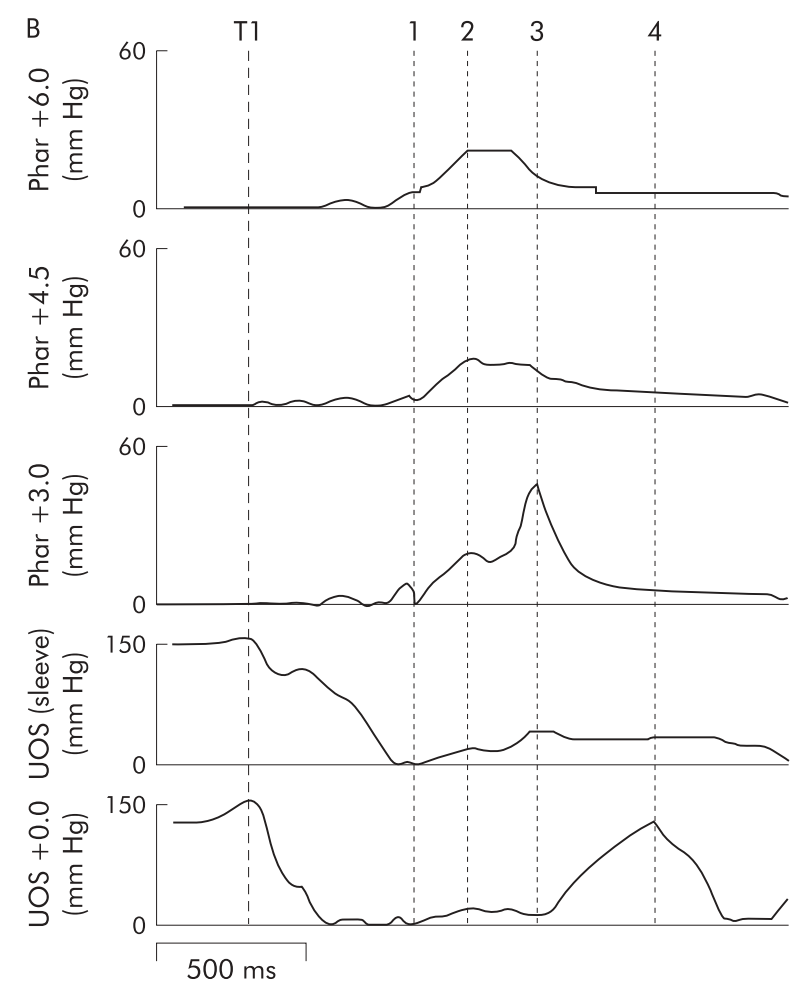

Figure 3 Videoradiographic sequence (A) and corresponding manometry (B) in a patient with myositis, a cricopharyngeal bar, and early diverticulum. Each vertical broken line in (B) represents the time corresponding to the numbered radiographic frame in (A). Note that the sphincter relaxes completely and there is a pharyngeal swallow response detected both radiographically and manometrically. Although the pharyngeal stripping wave is apparent, it is of low amplitude. Hypopharyngeal intrabolus pressure (frame 2, channel 3) is increased due to the marked restriction in sphincter opening. TI, onset of swallow indicated by initial tongue tip motion at the maxillary incisors. UOS, upper oesophageal sphincter. 

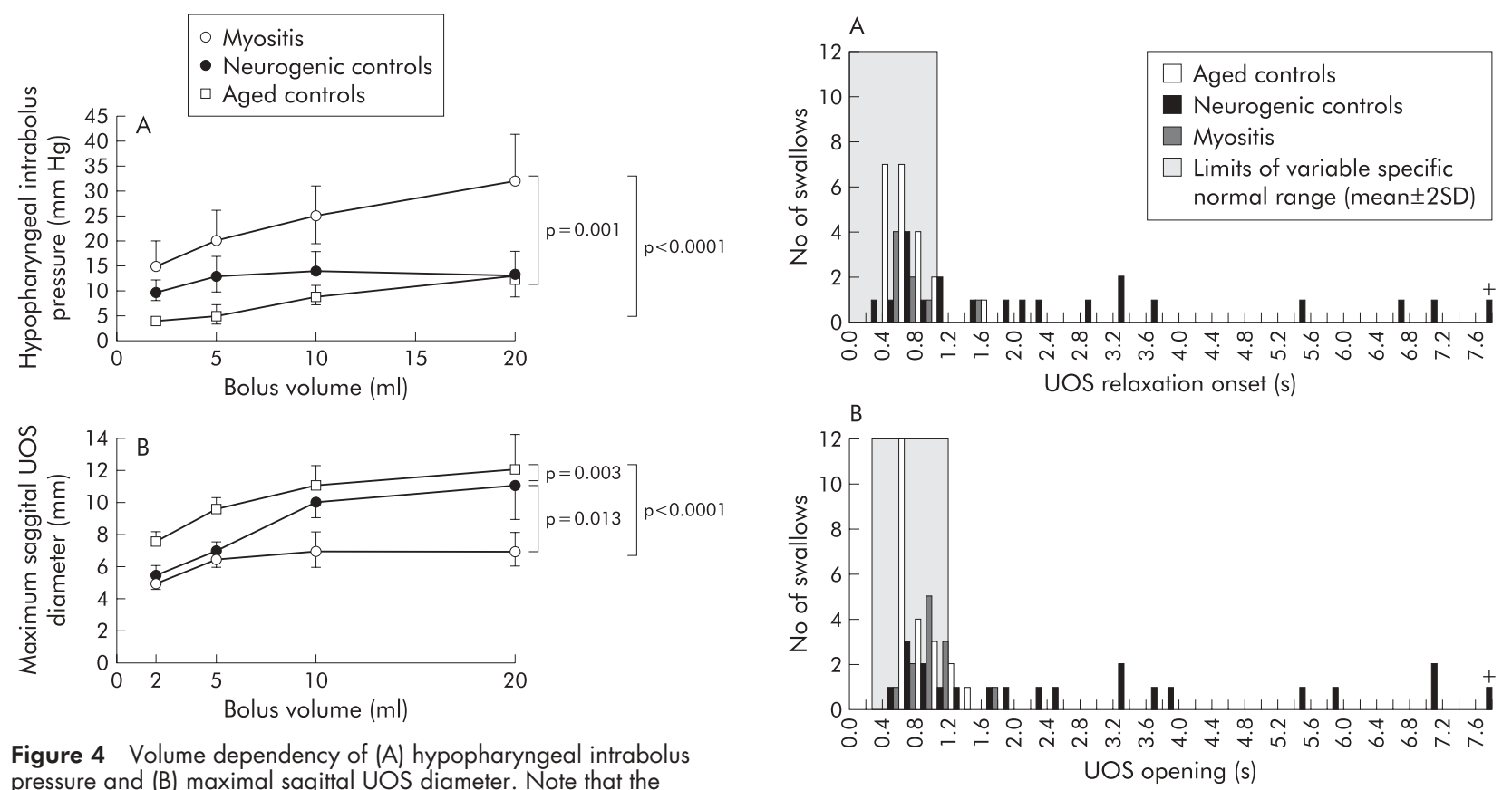

Figure 4 Volume dependency of (A) hypopharyngeal intrabolus extent of sphincter opening is reduced in myositis. Myositis patients also demonstrated a bolus volume dependent increase in hypopharyngeal intrabolus pressure which was significantly greater than that seen in either healthy or neurogenic controls. UOS, upper oesophageal sphincter.

(5) $\mathrm{mm} \mathrm{Hg}$ ) but was significantly greater than UOS pressure in neurogenic controls (25 (4); $\mathrm{p}=0.005$ ) (table 3 ). Nadir UOS pressure during dry swallows was normal in all but one patient (range -9 to $11 \mathrm{~mm} \mathrm{Hg}$ ). In one patient, who had severe UOS stenosis, deglutitive nadir UOS pressure was elevated (15 mm Hg).

Peak mid pharyngeal pressures were comparable between myositis patients (71 (7) $\mathrm{mm} \mathrm{Hg}$ ) and neurogenic controls (91 (14) $\mathrm{mm} \mathrm{Hg}$ ) but were significantly lower compared with aged healthy controls $(124(12) \mathrm{mm} \mathrm{Hg})(\mathrm{p}<0.05)$ (table 3). Six of 13 patients and 3/14 neurogenic controls demonstrated subnormal peak (hypopharyngeal) pressures (defined as $<57$ mm Hg; mean -2 SD in aged controls), implying significant pharyngeal weakness (fig 3).

\section{Biomechanical properties of the UOS}

To assess the biomechanical properties of the UOS, we examined the volume dependency of both hypopharyngeal intrabolus pressure and maximal UOS sagittal diameter (fig 4). Consistent with the qualitative impression of the high incidence of CP bar and stenosis, maximal sagittal UOS opening diameter was significantly reduced over the range of swallowed bolus volumes in patients with myositis $(\mathrm{p}<0.0001 v$ aged controls; $\mathrm{p}=0.013 v$ neurogenic controls) compared with both control groups (fig 4A). Myositis patients demonstrated a bolus volume dependent increase in hypopharyngeal intrabolus pressure over the range of swallowed bolus volumes, which was significantly greater than that seen in either healthy or neurogenic controls (fig 4B).

\section{Temporal measures in the swallow sequence: swallow coordination}

The distributions of individual timing intervals for patients and both control groups are shown in fig 5 . The right shift indicates that, in neurogenic controls, there was a substantial delay in one or more of sphincter (manometric) relaxation, sphincter (radiographic) opening, or sphincter closure. In contrast, the timing of UOS relaxation, opening, and closure in myositis is very close to that of healthy controls. An example of this delay in UOS relaxation can be seen in fig 6 .

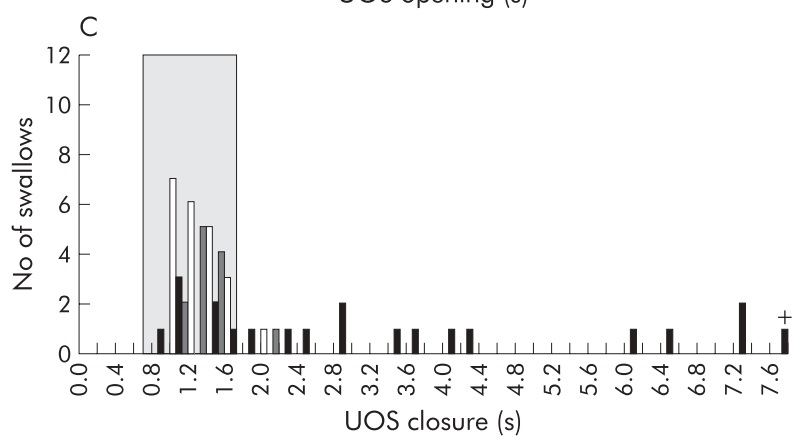

Figure 5 Swallow coordination in myogenic compared with neurogenic dysphagia. Shown are the timing of three major swallow events: upper oesophageal sphincter (UOS) relaxation onset (A), UOS opening (B), and UOS closure (C). Times are referenced to the onset of the oral phase, marked by initial motion of the tongue tip against the maxillary incisors. The shaded segments represent the normal range for healthy controls (mean $\pm 2 \mathrm{SD}$ ). Note that the timing of these swallow events in those with myogenic dysphagia is clustered very closely with that of healthy aged controls. The timing of these events in a large number of patients with neurogenic dysphagia falls well outside the normal range. + , one neurogenic control in whom timing of sphincteric events occurred at $+30-32$ seconds.

\section{Treatment and outcomes}

Ten of 13 cases were treated with prednisone. In addition, three patients in this group were treated with methotrexate, two with azathioprine, and one with cyclosporine. Three patients had medical treatment commenced at the time of dilatation, two commenced treatment more than 12 months prior, and one more than 12 months after $\mathrm{CP}$ disruption.

The overall 12 month mortality was $31 \%(4 / 13)$. Over 12 months of follow up, three patients (two with no response to dilatation and one not dilated) developed aspiration pneumonia and required PEG feeding. Subsequently, two of these patients died. One patient with scleroderma succumbed to a hypertensive crisis and another died of a sigmoid diverticular abscess 12 weeks after commencing prednisone.

Eight of the nine cases with restrictive abnormalities of the pharyngo-oesophageal segment on videoswallow had CP disruption by dilatation. Four of these eight had subjective and objective responses at one month. One responder died two months after the procedure (of a sigmoid diverticular abscess) and two of the remaining three patients remained improved at 

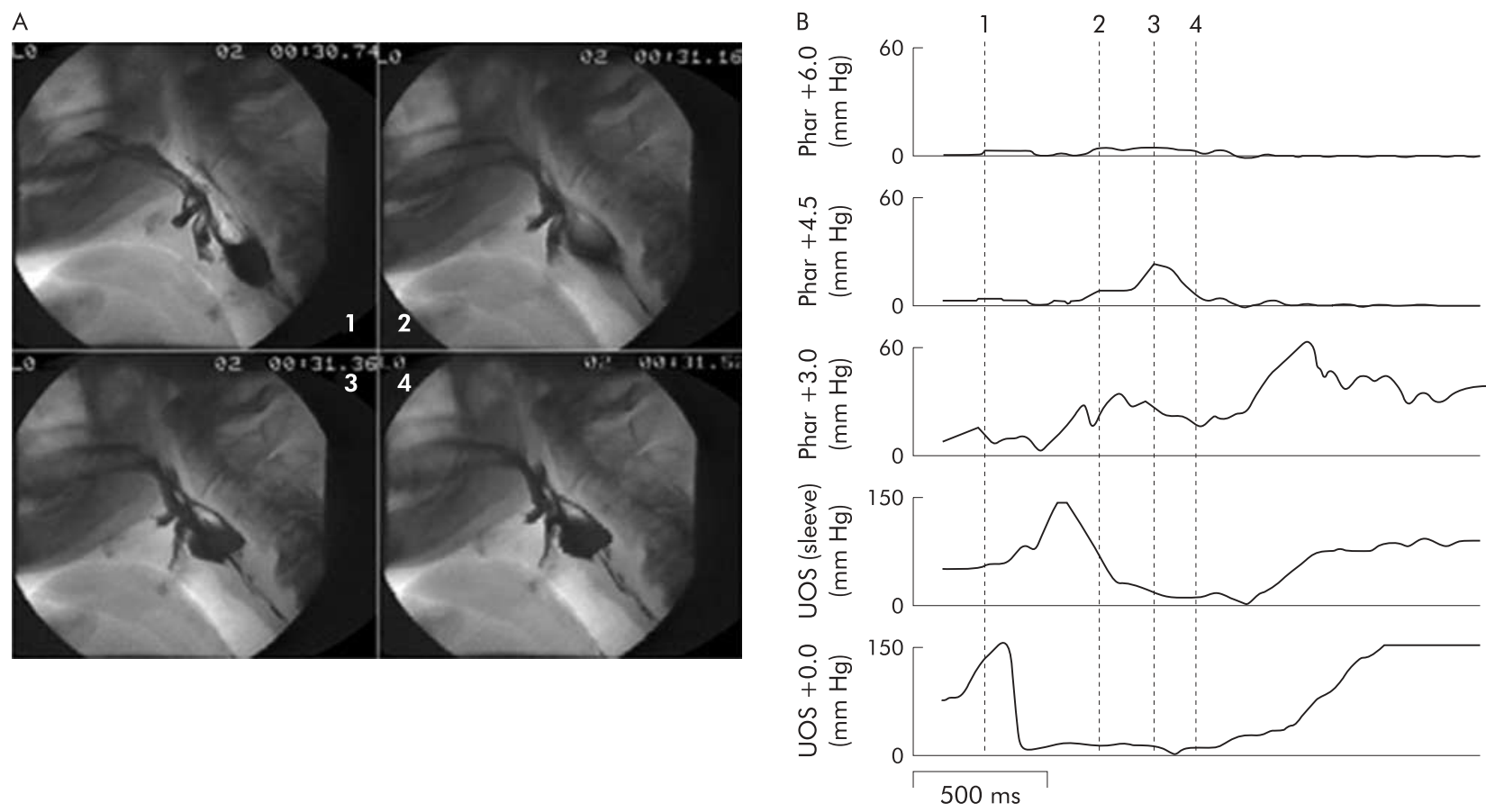

Figure 6 Videoradiographic frames $(A)$ and corresponding pharyngeal manometry $(B)$ in a disease control with neurogenic dysphagia due to brainstem stroke. The broken vertical lines in (B) correspond to the time of each of the four radiographs. Note the marked delay in onset of sphincter relaxation (channel 4) seen to occur after the onset of the hypopharyngeal pressure rise (channel 3). Compare this temporal relationship with fig 3 .

six months. Thus the overall short and long term response rates to dilatation were $50 \%$ and $25 \%$, respectively. The two long term responders, a patient that had an unsustained response and a patient who did not respond to dilatation, subsequently underwent CP myotomy, with further subjective and objective responses in the two that had previously responded to dilatation. Overall, an objective long term response to $\mathrm{CP}$ disruption was present in $3 / 8$ patients $(37.5 \%)$.

\section{DISCUSSION}

Inflammatory myopathy should be considered in any patient with otherwise unexplained oropharyngeal dysphagia. Inflammatory myopathy is a relatively uncommon aetiology for oropharyngeal dysphagia. In the present study, muscle disorders accounted for $5.7 \%$ of 529 cases of oropharyngeal dysphagia assessed at our institution over eight years. Two thirds of these muscle disorders (3.6\% of the total) were due to inflammatory myopathy. However, detecting this potentially treatable subgroup of patients with oropharyngeal dysphagia is an important clinical goal.

Our findings highlight some of the difficulties in making a diagnosis of myositis in cases presenting with dysphagia. One third of cases had no facial, bulbar, or limb weakness. This lack of physical findings very likely contributed to the substantial time between the onset of dysphagia and diagnosis (mean 55 months, up to 180 months). Other authors have reported cases in which dysphagia was the only detectable clinical manifestation of myopathy despite thorough neurological review. ${ }^{70}$

The other probable contributor to delay in diagnosis is the substantial false negative rate of laboratory tests. In the present study the diagnostic sensitivity of serum CPK was $75 \%$, which is comparable with a reported sensitivity of $70 \%$ in patients presenting with the full range of symptoms rather than dysphagia. ${ }^{7}$ Because of its high positive predictive value, a CPK level is one of the recommended screening tests in any new case of pharyngeal dysphagia of uncertain aetiology. ${ }^{2}$ However, the false negative rate of $25-30 \%$ needs to be kept in mind and mandates additional laboratory testing and/or muscle biopsy in suspected cases. ESR and ANA have poor diagnostic accuracy in this context. However, the sensitivity of one or more (of CPK, ESR, ANA) being abnormal was $100 \%$ in this study. Hence given the observed delay in diagnosis, the frequent lack of limb muscle weakness, and the difficulties in diagnosing myositis in the dysphagic population, it would seem appropriate to include ESR and ANA in the initial screening of suspected cases. The sensitivity of $71 \%$ for EMG in the present study was slightly lower than the $85-90 \%$ reported by others. ${ }^{7}$ It is possible that some of those presenting with dysphagia have a more focal form of the disease with more patchy limb muscle involvement and that specific sampling of head and neck muscles might improve the accuracy of EMG. For example, just under $25 \%$ of our patients had no apparent facial, bulbar, or limb muscle weakness on clinical examination. Others have also reported inflammatory changes apparently confined to the cricopharyngeus muscle. ${ }^{20} 23$ Also, EMG interpretation is highly operator dependent with relatively low inter-rater reliability. ${ }^{24}$ In view of the significant false negative rates for clinical examination and individual laboratory studies, a combination of CPK, EMG, and muscle biopsy should probably be performed in cases with dysphagia and suspected inflammatory myopathy or otherwise unexplained oropharyngeal dysphagia.

Restrictive CP abnormalities, either CP bar, stenosis, or Zenker's, were evident radiographically in $69 \%$ of cases of inflammatory myopathy. Although a CP bar is a common finding in normal individuals, there are sporadic reports of an apparent association between the CP bar and myositis. ${ }^{2022} 23$ Perhaps this reflects involvement of the pharyngeal musculature and $\mathrm{CP}$ in the myopathic process. Indeed, three of four patients undergoing CP myotomy in the present study had operative biopsy of the CP. In all three, inflammatory changes were observed in the CP muscle itself. Similar, but apparently focal, CP inflammatory myopathic findings have been reported by others. ${ }^{20223}$ We speculate that longstanding inflammation of the CP muscle may reduce its compliance thereby impeding the ability of the sphincter to open fully and thus impede transphincteric bolus flow. The higher than expected prevalence of Zenker's diverticula in this group may 
have pathophysiological importance. There has been only one other such case reporting this possible association. ${ }^{26} \mathrm{It}$ is now well established that the diverticulum is associated with a restrictive disorder of the $\mathrm{CP}^{11}{ }^{12}$ secondary to myonecrosis and fibroadipose tissue replacement within the muscle. ${ }^{27}$ While the majority of those with Zenker's do not have muscle disease these observations suggest that focal or general myositis, possibly through fibrogenesis within the $\mathrm{CP}$, might lead to the development of a Zenker's diverticulum in a small number of susceptible individuals.

We had hypothesised that compared with cases of neurogenic dysphagia, the full repertoire of swallow motor events should be preserved (albeit weaker) in myopathic dysphagia, and that phenomena which are driven by the medullary swallow centre (UOS relaxation, normal temporal relationships among events, and the pharyngeal swallow response) would be more likely to be disturbed in neurogenic dysphagia. Failure of manometric UOS relaxation, a relatively rare condition, is primarily a brainstem disorder. ${ }^{13}$ The one case of a supranormal deglutitive nadir UOS pressure in the myositis group was observed in an individual with a particularly tight CP stenosis. We believe this represents an increase in the intrabolus pressure seen with passage of residual saliva through a very tight sphincter in which the sleeve is positioned, rather than a primary neurogenic dysfunction. Hence preservation of UOS relaxation does not rule out a neurogenic cause, but failed UOS relaxation in the absence of a structural stenosis is highly predictive of a CNS (usually brainstem) aetiology. ${ }^{13}$ In the present study, the pharyngeal swallow response was absent in approximately $25 \%$ of cases of neurogenic dysphagia while it was invariably intact in myositis patients. Similarly, marked swallow incoordination (fig 5) was confined to neurogenic controls. Hence the finding of an absent pharyngeal swallow response or delay in key temporal measures which define UOS relaxation or opening, all militate against a myopathic aetiology and favour neurogenic dysphagia. It is recognised that the action of suprahyoid muscles facilitates the onset of UOS opening. ${ }^{16}$ We cannot be certain that possible involvement of the suprahyoid muscles by inflammation might have influenced apparent UOS relaxation determined manometrically. However, we believe it unlikely as intraluminal UOS pressure was abolished in all but one patient during swallow.

It is already known that the presence of dysphagia in a patient with myositis infers a higher mortality. ${ }^{2}$ Our findings confirm the poor prognosis of this association. Immunosuppressive therapies and CP disruption were the mainstay of treatment in this study. Despite multidisciplinary care of such cases, $31 \%$ of patients in this study died of their disease within 12 months of diagnosis. The mortality relates mainly to respiratory complications. Large prospective randomised controlled studies of steroids in polymyositis and dermatomyositis have not been performed. Hence steroid therapy followed by other immunosuppressive agents is largely empirical but widely accepted. ${ }^{8}$ Dysphagia has been reported anecdotally to improve with steroids alone in a few cases. The evidence supporting $\mathrm{CP}$ disruption is even less conclusive. There are no published randomised controlled trials of $\mathrm{CP}$ dilatation or myotomy but the response rate for neuromyogenic dysphagia is $62 \%$ overall. ${ }^{21}$ Anecdotal reports suggest that patients with polymyositis, dermatomyositis, or inclusion body myositis may benefit from CP myotomy. ${ }^{3-62128}$ However, despite the high prevalence of restrictive $\mathrm{CP}$ disorders in the present study, the short term response rate following $\mathrm{CP}$ disruption was only $50 \%$ in this relatively small group.

\section{ACKNOWLEDGEMENTS}

Components of this work were reported in part at Digestive Diseases Week, May 2000, San Diego, California (published in abstract form in
Gastroenterology 2000;118(Suppl):A4768) and at the 17th International Symposium on Neurogastroenterology and Motility, September 1999, Brugge, Belgium (published in abstract form in Neurogastroenterol Mot 1999;11(Suppl):A301).

\section{Authors' affiliations}

R B Williams, M J Grehan, J Andre, I J Cook, Department of Gastroenterology, St George Hospital, University of New South Wales, Sydney, Australia

M Hersch, Department of Neurology, St George Hospital, University of New South Wales, Sydney, Australia

\section{REFERENCES}

1 Buchholz DW. Neurogenic Dysphagia: what is the cause when the cause is not obvious? Dysphagia 1994;9:245-55

2 Marie I, Hatron PY, Levesque $\mathrm{H}$, et al. Influence of age on characteristics of polymyositis and dermatomyositis in adults. Medicine 1999:78:139-47.

3 Dietz F, Logeman JA, Sahgal V, et al. Cricopharyngeal muscle dysfunction in the differential diagnosis of dysphagia in polymyositis. Arthritis Rheum 1980;23:491-5.

4 Thomas F, LeBauer S, Greenberger N. Polymyositis masquerading as carcinoma of the cervical oesophagus. Arch Intern Med 1972; 129:984-6

5 Porubsky ES, Murray JP, Pratt LL. Cricopharyngeal achalasia in dermatomyositis. Arch Otolaryngol 1973;78:428-9.

6 Kagen L, Hochman R, Strong E. Cricopharyngeal obstruction in inflammatory myopathy (polymyositis/dermatomyositis). Arthritis Rheum 1985;28:630-6.

7 Tymms KE, Webb J. Dermatopolymyositis and other connective tissue diseases: a review of 105 cases. J Rheumatol 1985;12:1140-8.

8 Dalakas MC. Polymyositis, dermatomyosotis and inclusion body myositis. N Engl J Med 1991;325:1487-98.

9 Kleckner FS. Dermatomyositis and its manifestations in the gastrointestinal tract. Am J Gastroenterol 1970;53:141-6.

10 Dantas RO, Cook IJ, Dodds WJ, et al. Biomechanics of cricopharyngeal bars. Gastroenterology 1990;99:1269-74.

11 Cook IJ, Gabb M, Panagopoulos V, et al. Pharyngeal (Zenker's) diverticulum is a disorder of upper esophageal sphincter opening. Gastroenterology 1992;103:1229-35.

12 Shaw DW, Cook IJ, Jamieson GG, et al. Influence of surgery on deglutitive upper esophageal sphincter mechanics in Zenker's diverticulum. Gut 1996;38:806-11.

13 Williams RB, Wallace KL, Ali GN, et al. Biomechanics of failed deglutitive upper esophageal sphincter (UES) relaxation in patients with neurogenic dysphagia. Am J Physiol Gastrointest Liver Physiol neurogenic dysphag

14 Ali GN, Wallace KL, Schwartz R, et al. Mechanisms of oral-pharyngeal dysphagia in patients with Parkinson's disease. Gastroenterology 1996:110:383-92

15 Cook IJ. Disorders causing oropharyngeal dysphagia. In: Castell DO Richter JE, eds. The esophagus, 3rd edn. Philadelphia: Lippincott Williams and Wilkins, 1999:165-84.

16 Cook IJ, Dodds WJ, Dantas RO, et al. Opening mechanisms of the human upper esophageal sphincter. Am J Physiol 1989;257:G748-59.

17 Shaw DW, Cook IJ, Gabb M, et al. Influence of normal aging on oral-pharyngeal and upper esophageal sphincter function during swallowing. Am J Physiol 1995:268:G389-96.

18 Ali GN. Afferent and Efferent Control of the Oral-Pharyngeal Swallow (MD). Sydney: University of NSW, 1997

19 Bruning JL, Kintz BL. Computational Handbook of Statistics 2nd edn. Illinois: Scott, Foresman and Company, 1977

20 Shapiro J, Martin S, DeGirolami U, et al. Inflammatory myopathy causing pharyngeal dysphagia: a new entity. Ann Otol Rhinol Laryngol 1996:105:331-5.

21 Cook IJ, Kahrilas PJ. American Gastroenterological Association technical review on management of oropharyngeal dysphagia. Gastroenterology $1999 ; 116: 455-78$

22 Horvath OP, Zombari J, Halmos L, et al. Pharyngeal dysphagia caused by isolated myogen dystrophy of musculus cricopharyngeus. Acta Chir Hung 1998:37:51-8.

23 Bachmann G, Streppel M, Krug B, et al. Cricopharyngeal muscle hypertrophy associated with florid myositis. Dysphagia $2001 ; 16: 244-8$

24 Johnsen B, Juglsang-Frederiksen A, Vingtoft S, et al. Inter- and intraobserver variationin the interpretation of electromyographic tests. Electromyogr Clin Neurophysiol 1995;1995:432-43.

25 Curtis DJ, Cruess DF, Berg T. The cricopharyngeal muscle: a videorecording review. AJR Am J Roentgenol 1984;142:497-500.

26 Georgalas C, Baer ST. Pharyngeal pouch and polymyositis: association and implications for aetiology of Zenker's diverticulum. J Laryngol Otol 2000; 1 14:805-7

27 Cook IJ, Blumbergs $\mathrm{P}$, Cash K, et al. Structural abnormalities of the cricopharyngeus muscle in patients with pharyngeal (Zenker's) diverticulum. I Gastroenterol Hepatol 1992; 7:556-62.

28 Vencoksky J, Rehak F, Pafko P, et al. Acute cricopharyngeal obstruction in dermatomyositis. J Rheumatology 1088;15:1016-18. 